\title{
Out-of-plane Coupling Structures for Optical Printed Circuit Boards
}

\author{
N. Hendrickx ${ }^{1}$, J. Van Erps ${ }^{2}$, H. Thienpont ${ }^{2}$, P. Van Daele ${ }^{1}$ \\ ${ }^{1}$ Ghent University, INTEC-TFCG Microsystems, Technologiepark 914A, B-9052 Ghent, Belgium \\ ${ }^{2}$ Vrije Universiteit Brussel, Dept. of Applied Physics and Photonics (FirW-TONA), Pleinlaan 2, B-1050 Brussels, Belgium \\ E-mail: nina.hendrickx@intec.ugent.be
}

Abstract: We present an integrated total internal reflection mirror and pluggable coupler that can be used for out-of-plane coupling in an optical PCB. The coupling efficiency of both mirror configurations is measured and compared.

\section{Introduction}

Optical interconnections offer a possible solution to the bandwidth problems that are associated with electrical interconnections [1]. The integration of optical interconnects to the board-level, covering distances from a couple of centimeters to one meter, can be done with the use of a polymer optical layer applied on top of or laminated into the Printed Circuit Board (PCB). The optical layer contains multimode optical waveguides for in-plane light propagation. Coupling structures, which deflect the light beam over $90^{\circ}$, are required to couple the light from/to an optical transmitter/receiver to/from the waveguides. Compatibility with the existing PCB manufacturing and soldering processes is required to come to a cost-effective solution.

We present a $45^{\circ}$ mirror configuration which is directly integrated with the optical waveguides and a pluggable coupler which can be inserted into a cavity in the optical layer. The integrated mirror is patterned into the optical layer with use of laser ablation. The pluggable coupler is patterned into a PMMA substrate with use of Deep Proton Writing (DPW). Both mirror structures can be used as coupling elements for out-of-plane coupling in an optical PCB.

\section{Micro-structuring technologies}

Laser ablation is a flexible non-contact micro-structuring technology that is fully compatible with existing PCB manufacturing and soldering processes and is already used for the laser-drilling of micro-vias in high density electrical boards. A KrF excimer laser $(248 \mathrm{~nm})$ is used for the ablation of micro-optical components into the polymer optical layer [2]. Polymers show excellent absorption properties in the UV-range which makes a clean ablation possible, enabling us to pattern components with a low surface roughness. The KrF excimer laser beam can be tilted which allows us to ablate $45^{\circ}$ mirror facets in a very straightforward way. There is always a certain degree of tapering during the ablation which can however be measured and corrected.

Deep Proton Writing (DPW) [3] is a rapid prototyping technology that consists of a patterned 8.3MeV irradiation of a polymer photoresist (PMMA) followed by a selective etching process to remove the exposed zones. Although it is obvious that DPW is not a mass fabrication technique, one of its assets is that once the master component has been prototyped with DPW, a metal mould can be generated from the master by electroplating. After removal of the plastic master, this metal mould can be used as a shim in a final micro-injection moulding or hot embossing step. This way, the component can be mass-produced at low cost in a wide variety of high-tech plastics.

\section{Coupling structures}

The ablated $45^{\circ}$ micro-mirror is integrated with the optical waveguides, which allows us to obtain high alignment accuracy. The mirror facet is ablated into the optical layer with the tilted $\mathrm{KrF}$ excimer laser; the alignment with respect to the waveguides is done with the help of alignment marks. The $90^{\circ}$ beam deflection is based on the total internal reflection (TIR) at the polymer-air interface. The mirror facet can be Au-coated to protect it from environmental influence. The main advantage of this mirror configuration is the fact that the alignment between the waveguide and the mirror is arranged during the patterning; the main disadvantage is the requirement for compatibility with all subsequent processing steps. A scheme of this TIR mirror configuration and SEM images of experimental realizations are given in Fig. 1.

The pluggable coupler is patterned into a 500 $\mu \mathrm{m}$ thick PMMA substrate using DPW. It contains a $45^{\circ}$ mirror facet which is used for the $90^{\circ}$ beam deflection. The coupler is plugged into a micro-cavity which is ablated into the optical layer. Because of the tapering that occurs during the ablation, the cavity is ablated with a slightly tilted KrF excimer laser beam in order to obtain a vertical wall which can be used as output facet of the waveguides. The coupler is inserted in the cavity manually; a red light source is used to arrange the alignment of the coupler in the cavity. The insert can be placed in a very late phase of processing which makes the compatibility with the entire fabrication process less stringent. A scheme and practical realization of this DPW coupler are shown in Fig. 2. 


\section{JThA8.pdf}

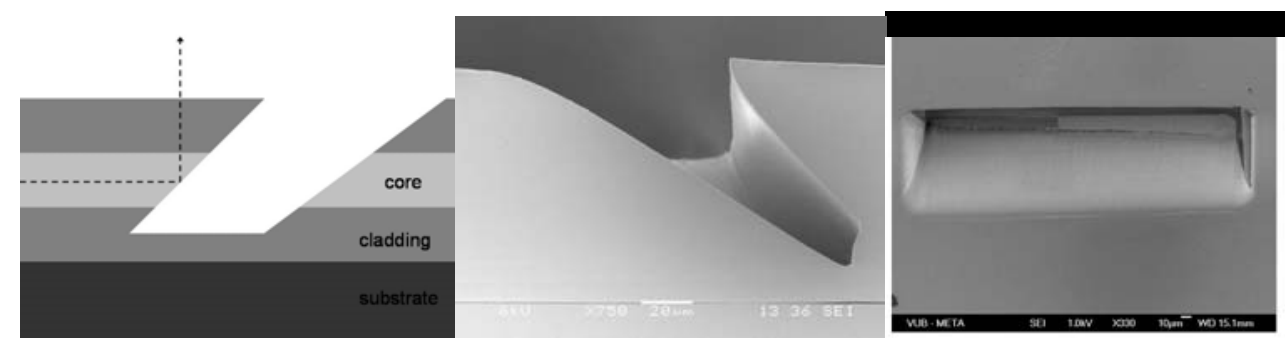

Figure 1: The left picture shows the principle of the TIR mirror; the middle picture is an SEM image of an ablated TIR mirror; the right picture shows a top view of an ablated TIR mirror.

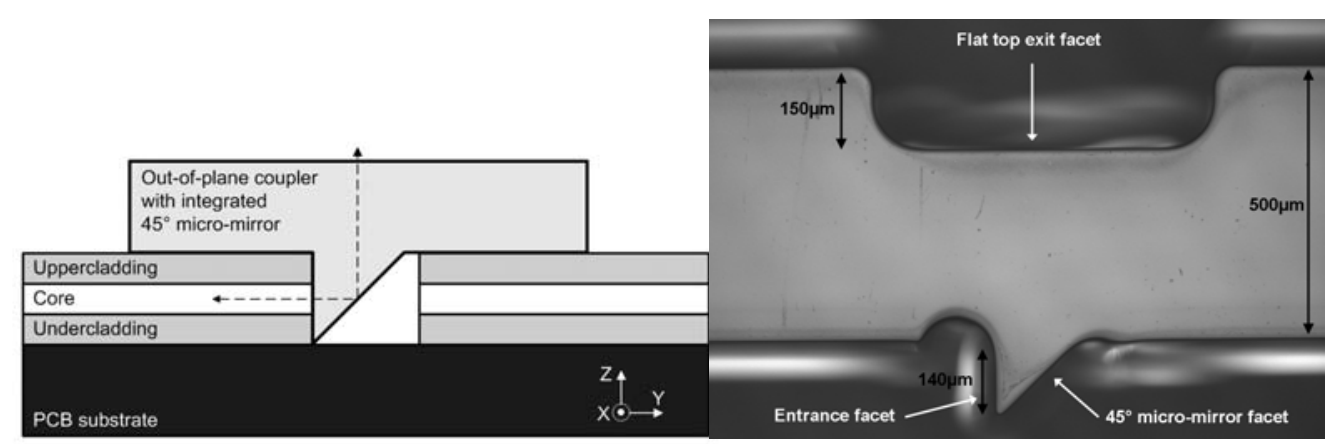

Figure 2: The left picture shows a schematic view of a pluggable coupler inserted in a micro-cavity; the right picture shows the pluggable DPW coupler.

4. Coupling efficiency measurements

Coupling efficiency measurements are performed for both the TIR mirror and the pluggable coupler on comparable samples. The optical layer, Truemode Backplane ${ }^{\mathrm{TM}}$ Polymer, is spin-coated on an FR4 substrate and contains laser ablated multimode optical waveguides with a cross-section of $50 \mu \mathrm{m} \times 50 \mu \mathrm{m}$ on a pitch of $125 \mu \mathrm{m}$ [4]. The waveguides have a numerical aperture (NA) of 0.3 and have a propagation loss of $0.12 \mathrm{~dB} / \mathrm{cm}$ at the launching wavelength of $850 \mathrm{~nm}$.

For the characterization of the mirrors in a receiver structure, we used a 50 $\mu \mathrm{m}$ core diameter silica multimode fiber (MMF) with NA 0.2 as input fiber and a $100 \mu \mathrm{m}$ core diameter silica MMF with 0.29 NA as output fiber. The sample is mounted on a sixaxis parallel kinematics motion robot, which allows us to both position the fiber with an accuracy of 300nm and perform two-axis scans to measure the tolerance for mechanical misalignments of the detector fiber. The reference measurement consists of an inline butt coupling of both fibers. For the case of the TIR mirror, the measured overall link loss (butt-coupling at the input facet of the waveguides, propagation through the waveguides, $90^{\circ}$ beam deflection at the TIR mirror and outcoupling towards the detector fiber) is $-2.55 \mathrm{~dB}$. The application of Au-coating on the TIR facet could improve this value. The lateral scan of the coupling efficiency is given in the left image of Fig. 3. For the case of the pluggable coupler, the component was manually placed into the laser ablated micro-cavity into the optical layer. The length of the ablated waveguides on this sample is approximately $5 \mathrm{~cm}$ as opposed to the sample with the TIR mirror where the waveguide length is only $2 \mathrm{~cm}$. The measured overall link loss is $5.96 \mathrm{~dB}$. The lateral scan of the coupling efficiency is given in the right part of Fig. 3. The use of index matching gel and Aucoating of the mirror facet could improve the measured efficiency. In case the input and output fiber are placed at the input and output facet of the pluggable coupler, the measured coupling efficiency is $-0.77 \mathrm{~dB}$. This indicates that it should be possible to improve the measured efficiency on the overall link. Fig. 4 shows a picture of both experimental set-up configurations.

For the characterization of the mirrors in a transmitter structure, we used a SMF-28 fiber with NA 0.13 to couple the light in vertically towards the mirror. The low NA of the SMF will in this case ensure that no light is lost at the mirror due to not satisfying the TIR condition. The light spot that is coupled out by the waveguides in-plane is detected with a $100 \mu \mathrm{m}$ diameter MMF with NA 0.29 . The measured coupling efficiency is $-1.59 \mathrm{~dB}$ for the TIR mirror sample and -3.00dB for the sample with the pluggable coupler. 


\section{JThA8.pdf}
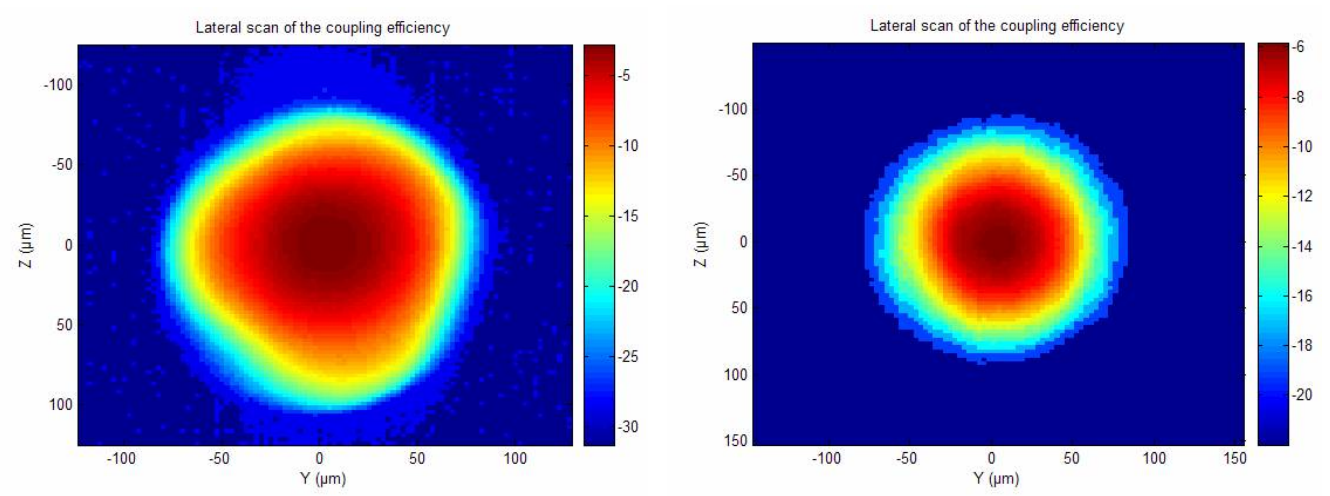

Figure 3: Lateral scan of the coupling efficiency $(\mathrm{dB})$ of a complete link where a TIR mirror (left) or pluggable coupler (right) is used for the out-of-plane coupling.
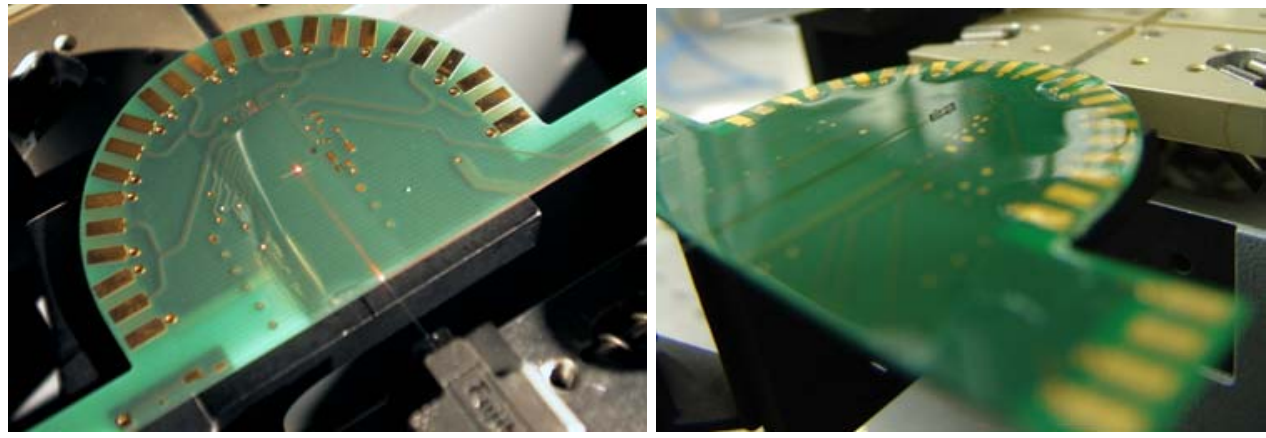

Figure 4: The left picture shows a red light spot coupled out at an ablated TIR mirror; the right picture shows an optical PCB where the pluggable coupler is inserted into a laser ablated cavity in the optical layer.

Conclusion

We presented two different coupling structures which can be used for out-of-plane coupling in an optical PCB. The first mirror configuration is a laser ablated total internal reflection (TIR) mirror, which is patterned into the optical layer and is integrated with the optical waveguides. The second structure is a pluggable coupler which contains a TIR mirror and which can be plugged into an ablated micro-cavity in the optical layer either manually or with pick-and-place equipment. The coupling efficiency at $850 \mathrm{~nm}$ of both mirrors has been measured for both the situation of a transmitter and receiver side of the board. The coupling efficiencies at the receiver side are lower than the ones at the transmitter side. This is mainly caused by the high NA of the waveguides; a certain number of rays will not satisfy the TIR condition at the mirror facet and will for this reason not contribute to the detected light signal. For the measurements at the transmitter side, the low NA of the SMF that is used to couple the light signal into the mirror will ensure that no light is lost at the mirror due to not satisfying the TIR condition.

Acknowledgements

N. Hendrickx would like to acknowledge the Institute for the Promotion of Innovation by Science and Technology in Flanders (IWT Flanders) for financial support. J. Van Erps acknowledges the FWO for financial support. Part of this work was carried out within the framework of the Network of Excellence on Micro-Optics (NEMO), supported by the European Commission through FP6 program and in the framework of the FAOS project (Flexible Artificial Optical Skin) supported by the Institute for the Promotion of Innovation by Science and Technology in Flanders (IWT Flanders).

\section{References}

[1] D. A. B. Miller, "Rationale and challenges for optical interconnects to electronic chips,” Proc. IEEE, vol. 88, no. 6, pp. 728-749, Jun. 2000.

[2] G. Van Steenberge et al., MT-compatible laser ablated interconnections for optical printed circuit boards, J. Lightwave Technology, Vol.22, no. 9, pp. 2083-2090

[3] C. Debaes et al., Deep Proton Writing: a rapid protoyping polymer microfabrication tool for micro-optical modules, New J. Phys., vol. 8, pp. 270-288, 2006.

[4] G. Van Steenberge et al, Laser ablation of parallel optical interconnect waveguides, Photon. Technol. Lett., vol. 18, no. 9, pp. 1106-1108, May 2006. 